\title{
On Weyl channels being covariant with respect to the maximum commutative group of unitaries
}

\author{
Grigori G. Amosov* \\ Department of Higher Mathematics \\ Moscow Institute of Physics and Technology \\ Dolgoprudny 141700 \\ RUSSIA
}

July 16, 2018

\begin{abstract}
We investigate the Weyl channels being covariant with respect to the maximum commutative group of unitary operators. This class includes the quantum depolarizing channel and the "two-Pauli" channel as well. Then, we show that our estimation of the output entropy for a tensor product of the phase damping channel and the identity channel based upon the decreasing property of the relative entropy allows to prove the additivity conjecture for the minimal output entropy for the quantum depolarizing channel in any prime dimesnsion and for the "two Pauli" channel in the qubit case.
\end{abstract}

\section{Introduction}

Let $H$ be a finite-dimensional Hilbert space. Denote $\sigma(H)$ the set of states (positive unit-trace operators) in $H$. The linear map $\Phi$ :

*E-mail: gramos@mail.ru 
$\sigma(H) \rightarrow \sigma(H)$ is said to be a quantum channel if $\Phi^{*}$ is completely positive $([8])$. The quantum channel $\Phi$ is called bistochastic if $\Phi\left(\frac{1}{d} I_{H}\right)=$ $\frac{1}{d} I_{H}$, where $I_{H}$ is the identity operator in $H$.

The Holevo-Schumacher-Westmoreland bound $C_{1}(\Phi)$ of a quantum channel $\Phi$ is defined by the formula

$$
C_{1}(\Phi)=\sup _{x_{j} \in \sigma(H), \pi} S\left(\sum_{j=1}^{r} \pi_{j} \Phi\left(x_{j}\right)\right)-\sum_{j=1}^{r} \pi_{j} S\left(\Phi\left(x_{j}\right)\right),
$$

where $S(x)=-\operatorname{Tr} x \log x$ is the von Neumann entropy of $x$ and the supremum is taken over all probability distributions $\pi=\left(\pi_{j}\right)_{j=1}^{r}, 0 \leq$ $\pi_{j} \leq 1, \sum_{j=1}^{r} \pi_{j}=1$ for all $r \in \mathbb{N}$. The additivity conjecture states (1]) that for any two channels $\Phi$ and $\Psi$

$$
C_{1}(\Phi \otimes \Psi)=C_{1}(\Phi)+C_{1}(\Psi)
$$

If the additivity conjecture holds, one can easily find the capacity $C(\Psi)$ of the channel $\Psi$ by the formula $C(\Psi)=\lim _{n \rightarrow+\infty} \frac{C_{1}\left(\Psi^{\otimes n}\right)}{n}=C_{1}(\Psi)$ (see [9]).

At the moment, the additivity conjecture is proved for a number of different cases $(9,13,14,16,5,3])$. The crucial role was played by C. King who proved [13, 14, the additivity conjecture for all bistochastic qubit channels as well as for the quanum depolarizing channel. In 2 it was shown that the additivity conjecture for the channels of the form $\Psi \circ \Phi$, where $\Phi$ is the quantum depolarizing channel and $\Psi$ is the phase damping can be proved by means of the decreasing property of the relative entropy [15]. In the present paper we continue to study a possibility to use the decreasing property of the relative entropy to estimate the von Neumann entropy of the output of a quantum channel.

The additivity conjecture for the quantity $C_{1}$ is closely connected with the additivity conjecture for the entropy infimum which states that

$$
\inf _{\rho \in \sigma(H \otimes K)} S((\Phi \otimes \Psi)(\rho))=\inf _{\rho \in \sigma(H)} S(\Phi(\rho))+\inf _{\rho \in \sigma(K)} S(\Psi(\rho))
$$

for any two channels $\Phi$ and $\Psi$ acting in Hilbert spaces $H$ and $K$, respectively. For the case of the Weyl channel we consider in the present paper, if the additivity conjecture for the entropy infimum 
holds it implies that the quantity $C_{1}$ is additive also ([10]). As it is shown in [17] the additivity conjectures for $C_{1}$ and the entropy infimum are globally equivalent.

Fix the basis $e_{j}, 0 \leq j \leq d-1$, in a Hilbert space $H, \operatorname{dim} H=d$, and define a unitary operator $U$ by the formula

$$
U=\sum_{j=0}^{d-1} e^{\frac{2 \pi i}{d} j}\left|e_{j}><e_{j}\right|
$$

Given a probability distribution $0 \leq \lambda_{j} \leq 1, \sum_{j=0}^{d-1} \lambda_{j}=1$, we shall call the bistochastic quantum channel defined as

$$
\Phi(x)=\sum_{j=0}^{d-1} \lambda_{j} U^{j} x U^{* j}, x \in \sigma(H),
$$

by a phase damping. The map

$$
E(x)=\frac{1}{d} \sum_{j=0}^{d-1} U^{j} x U^{* j} \equiv \sum_{j=0}^{d-1}\left|e_{j}><e_{j}\right| x\left|e_{j}><e_{j}\right|,
$$

$x \in \sigma(H)$, is a conditional expectation to the algebra of the elements being fixed with respect to the action of the group $\left\{U^{j}, 0 \leq j \leq d-1\right\}$ and, hence, of the channel $\Phi$.

Given two states $\rho$ and $\bar{\rho}$, let $S(\rho, \bar{\rho})=\operatorname{Tr}(\rho \log \rho)-\operatorname{Tr}(\rho \log \bar{\rho})$ be the Umegaki relative entropy [18. Due to [15] the following property holds,

$$
S(\Phi(\rho), \Phi(\bar{\rho})) \leq S(\rho, \bar{\rho})
$$

for any (not necessarily bistochastic) quantum channel $\Phi$. Using this property it is possible to estimate the entropy of the output of a quantum channel ([2]).

Let $K$ be an arbitary finite-dimensional Hilbert space. Using the decreasing property of the relative entropy we shall prove the following statement.

Theorem 1.Let $x \in \sigma(H \otimes K)$ be such that

$$
E\left(\operatorname{Tr}_{K}(x)\right)=\frac{1}{d} I_{H}
$$

Then, for the phase damping (1) we get

$$
S((\Phi \otimes I d)(x)) \geq-\sum_{j=0}^{d-1} \lambda_{j} \log \lambda_{j}+\frac{1}{d} \sum_{j=0}^{d-1} S\left(x_{j}\right),
$$


where $x_{j}=d \operatorname{Tr}_{H}\left(\left(\left|e_{j}><e_{j}\right| \otimes I_{K}\right) x\right) \in \sigma(K), 0 \leq j \leq d-1$.

Analyzing the Weyl channels being covariant with respect to the maximum commutative group of unitary operators we shall prove the following two theorems based upon the estimation (2).

Theorem 2.Let $\Phi(\rho)=(1-p) \rho+\frac{p}{d} I_{H}, \rho \in \sigma(H), 0 \leq p \leq \frac{d^{2}}{d^{2}-1}$, be the quantum depolarizing channel in the Hilbert space $H$ of the prime dimension $d$. Then, there exists d orthonormal bases $\left\{f_{j}^{s}, 0 \leq\right.$ $s, j \leq d-1\}$ in $H$ such that

$$
\begin{gathered}
S((\Phi \otimes I d)(x)) \geq-\left(1-\frac{d-1}{d} p\right) \log \left(1-\frac{d-1}{d} p\right)-\frac{d-1}{d} p \log \frac{p}{d}+ \\
\frac{1}{d^{2}} \sum_{j=0}^{d-1} \sum_{s=0}^{d-1} S\left(x_{j}^{s}\right),
\end{gathered}
$$

where $x \in \sigma(H \otimes K), x_{j}^{s}=d \operatorname{Tr}_{H}\left(\left(\left|e_{j}^{s}><e_{j}^{s}\right| \otimes I_{K}\right) x\right) \in \sigma(K), 0 \leq$ $j, s \leq d-1$.

Denote $\sigma_{x}, \sigma_{y}$ and $\sigma_{z}$ the Pauli operators.

Theorem 3.Let $\Phi(\rho)=(1-2 p) \rho+p \sigma_{y} \rho \sigma_{y}+p \sigma_{z} \rho \sigma_{z}$ be the "two Pauli" channel in the space $H$, dim $H=2$. Suppose that $p \leq \frac{1}{3}$, then there exist three orthonormal bases $\left(e_{1}^{s}, e_{2}^{s}\right), 1 \leq s \leq 3$, in $H$ such that

$S((\Phi \otimes I d)(x)) \geq-(1-p) \log (1-p)-p \log p+\frac{1}{6} \sum_{n=1}^{3}\left(S\left(x_{j}^{1}\right)+S\left(x_{j}^{2}\right)\right)$,

where $x \in \sigma(H \otimes K), x_{j}^{k}=2 \operatorname{Tr}_{H}\left(\left(\left|e_{k}^{j}><e_{k}^{j}\right| \otimes I_{K}\right) x\right) \in \sigma(K), 1 \leq$ $j \leq 3,1 \leq k \leq 2$.

Remark. The additivity of the minimal output entropy for the quantum depolarizing channel and the "two-Pauli" channel follows from Theorems 2 and 3. Indeed, these theorems contain more information. It was shown in [13] that if the additivity conjecture holds for the qubit depolarizing channel and the "two-Pauli" channel in the case $p \leq \frac{1}{3}$, then it holds for all bistochastic qubit channels. Hence, in particular, Theorems 2 and 3 imply that the additivity conjecture holds for all bistochastic qubit channels.

This paper is organized as follows. In Part 2 we prove Theorem 1. Part 3 is devoted to the description of the Weyl (the same as Weylcovariant) channels. The orbits of the maximum commutative groups in the dimensions $d=2$ and $d=3$ are described in Part 3. In Part 4 we find the conditions which are sufficient for the Weyl channel be covariant with respect to the maximum commutative group. In Part 5 the proof of Theorems 2 and 3 is given. 


\section{Proof of Theorem 1}

In this section we shall use the approach introduced in [2].

Fix $x$ with the property formulated in Theorem 1 and define a quantum channel $\Xi_{x}: \sigma(H \otimes K) \rightarrow \sigma(H \otimes K)$ by the formula

$\Xi_{x}(\rho)=\sum_{j=0}^{d-1} \operatorname{Tr}\left(\left(\left|e_{j}><e_{j}\right| \otimes I_{K}\right) \rho\right)\left(U^{j} \otimes I_{K}\right) x\left(U^{* j} \otimes I_{K}\right), \rho \in \sigma(H \otimes K)$.

Put $\rho=\sum_{j=0}^{d-1} \lambda_{j}\left|e_{j}><e_{j}\right| \otimes y, \bar{\rho}=\sum_{j=0}^{d-1} \frac{1}{d}\left|e_{j}><e_{j}\right| \otimes y=\frac{1}{d} I_{H} \otimes y$, where $y \in \sigma(K)$ is an arbitrary fixed state. Then,

$$
\begin{gathered}
\Xi_{x}(\rho)=(\Phi \otimes I d)(x) \\
\Xi_{x}(\bar{\rho})=\frac{1}{d} \sum_{j=0}^{d-1}\left(U^{j} \otimes I_{K}\right) x\left(U^{* j} \otimes I_{K}\right) \equiv \tilde{E}(x) .
\end{gathered}
$$

Notice that $\tilde{E}=(E \otimes I d)$ is the conditional expectation to algebra of the elements being fixed with respect to the action of the cyclic group $\left\{U^{j} \otimes I_{K}, 0 \leq j \leq d-1\right\}$.

The decreasing property of the relative entropy [15] gives us the estimation:

$$
S\left(\Xi_{x}(\rho), \Xi_{x}(\bar{\rho})\right) \leq S(\rho, \bar{\rho})=\sum_{j=0}^{d-1} \lambda_{j} \log \lambda_{j}+\log (d) .
$$

On the other hand,

$$
\begin{gathered}
S\left(\Xi_{x}(\rho), \Xi_{x}(\bar{\rho})\right)=\operatorname{Tr}((\Phi \otimes I d)(x) \log (\Phi \otimes I d)(x))- \\
\operatorname{Tr}((\Phi \otimes I d)(x) \log \tilde{E}(x))=-S((\Phi \otimes I d)(x))- \\
\operatorname{Tr}(\tilde{E} \circ(\Phi \otimes I d)(x) \log \tilde{E}(x))= \\
-S((\Phi \otimes I d)(x))+S(\tilde{E}(x)) .
\end{gathered}
$$

Here we used the equality $\tilde{E} \circ(\Phi \otimes I d)=\tilde{E}$ which holds because $\tilde{E}$ is the conditional expectation to the algebra of elements being fixed with respect to the action of $\Phi \otimes I d$. It follows from the condition of Theorem 1 that

$$
\tilde{E}(x)=\frac{1}{d} \sum_{j=0}^{d-1}\left|e_{j}><e_{j}\right| \otimes x_{j}, x_{j} \in \sigma(K) .
$$


Thus,

$$
S(\tilde{E}(x))=\log (d)+\frac{1}{d} \sum_{j=0}^{d-1} S\left(x_{j}\right)
$$

$x_{j}=d \operatorname{Tr}_{H}\left(\left(\left|e_{j}><e_{j}\right| \otimes I_{K}\right) x\right), 0 \leq j \leq d-1$. Combining (44), (5) and (6) we get the result we need.

\section{The Weyl channels}

Recently the representation of quantum channels by means of the discrete Weyl group were discussed in different contexts ( 2, 4, 7, 12]). Fix the orthonormal basis $\mid k>, k=0,1, \ldots, d-1$ of the Hilbert space $H, \operatorname{dim} H=d$, and define the unitary operators $U_{m, n}$ by the formula ([6])

$$
U_{m, n}=\sum_{k=0}^{d-1} e^{\frac{2 \pi i}{d} k n}|k+m \bmod d><k|,
$$

$0 \leq m, n \leq d-1$. The operators $U_{m, n}$ are satisfied the Weyl commutation relations,

$$
U_{m, n} U_{m^{\prime}, n^{\prime}}=e^{2 \pi i\left(m^{\prime} n-m n^{\prime}\right) / d} U_{m^{\prime}, n^{\prime}} U_{m, n},
$$

$0 \leq m, n, m^{\prime}, n^{\prime} \leq d-1$. We shall call $U_{m, n}$ by a Weyl operator. Notice that

$$
\begin{gathered}
U_{m, 0}|k>=| k+m \bmod d>, U_{0, n}\left|k>=e^{\frac{2 \pi i}{d} k n \bmod d}\right| k>, \\
U_{m, n}=U_{m} U_{n}, 0 \leq m, n \leq d-1 .
\end{gathered}
$$

We shall consider bistochastic quantum communication channels of the following form

$$
\Phi(x)=\sum_{m, n=0}^{d-1} \pi_{m, n} U_{m, n} x U_{m, n}^{*},
$$

$x \in \sigma(H)$, where $0 \leq \pi_{m, n} \leq 1, \sum_{m, n=0}^{d-1} \pi_{m, n}=1$, is an arbitrary probability distribution. We shall call (9) by a Weyl channel.

The bases $\left(e_{j}\right)_{j=0}^{d-1}$ and $\left(f_{j}\right)_{j=0}^{d-1}$ of the space $H$ are said to be mutually unbiased bases ([1]) if $\left|<e_{j}\right| f_{k}>\mid=\frac{1}{\sqrt{d}}, 0 \leq j, k \leq d-1$. For 
example, the eigenvectors $f_{j}=\mid j>$ and $e_{j}=\frac{1}{\sqrt{d}} \sum_{k=0}^{d-1} e^{\frac{2 \pi i}{d} j k} \mid k>$ of the Weyl operators $U_{0, n}$ and $U_{m, 0}$, respectively, form mutually unbiased bases. If the dimension $d$ of the Hilbert space $H$ is prime, there exist $d+1$ mutually unbiased bases $\left\{e_{j}^{k}, 0 \leq j \leq d-1,0 \leq k \leq d\right\}$ which can defined as the eigenvectors of the Weyl operators $U_{s k, k}$ such that

$$
\begin{gathered}
\left|e_{j}^{s}><e_{j}^{s}\right|=\frac{1}{d} \sum_{k=0}^{d-1} e^{\frac{2 \pi i}{d} j k} U_{s k, k}, 0 \leq s \leq d-1, \\
\left|e_{j}^{d}><e_{j}^{d}\right|=\frac{1}{d} \sum_{k=0}^{d-1} e^{\frac{2 \pi i}{d} j k} U_{k, 0} \equiv|j><j| .
\end{gathered}
$$

Because the elements of the mutually unbiased bases $\left(e_{j}^{s}\right)$ are eigenvectors for the Weyl operators $U_{s k, k}$ and $U_{k, 0}$, we get

$$
\begin{gathered}
U_{s k, k}=\sum_{j=0}^{d-1} e^{\frac{2 \pi i}{d} j k}\left|e_{j}^{s}><e_{j}^{s}\right|, 0 \leq s \leq d-1, \\
U_{k, 0}=\sum_{j=0}^{d-1} e^{\frac{2 \pi i}{d} j k}\left|e_{j}^{d}><e_{j}^{d}\right| .
\end{gathered}
$$

Fix a basis $\left(e_{j}\right)_{j=0}^{d-1}$ in the space $H$. We shall call by the maximimum commutative group of unitaries $\mathcal{U}_{d}\left(\left(e_{j}\right)_{j=0}^{d-1}\right)$ a $d$-parameter group of unitary operators

$$
U=\sum_{j=0}^{d-1} e^{i \phi_{j}}\left|e_{j}><e_{j}\right|
$$

where $\phi_{j}, 0 \leq j \leq d-1$, are arbitrary real numbers. Denote by $\mathcal{U}_{d}^{s}=\mathcal{U}_{d}\left(\left(e_{j}^{s}\right)_{j=0}^{d-1}\right), \quad 0 \leq s \leq d$, the maximum commutative groups generated by the mutually unbiased bases (10).

The bistochastic quantum channel

$$
E_{s}(x)=\sum_{j=0}^{d-1}\left|e_{j}^{s}><e_{j}^{s}\right| x\left|e_{j}^{s}><e_{j}^{s}\right|, x \in \sigma(H),
$$

is a conditional expectation to the algebra of elements being fixed with respect to the action of the maximum commutative group $\mathcal{U}_{d}^{s}$ as well 
as of the phase damping channel

$$
\Phi_{s}(x)=\sum_{j=0}^{d-1} \lambda_{j} U_{s j, j} x U_{s j, j}^{*}
$$

for $0 \leq s \leq d-1$ and

$$
\Phi_{d}(x)=\sum_{j=0}^{d-1} \lambda_{j} U_{j, 0} x U_{j, 0}
$$

for $s=d$.

\section{Orbits of the maximum commuta- tive group of unitaries.}

Denote by $\mathcal{G}_{d}^{s}$ the set consisting of the unit vectors $g$ for which there exists the unitary operator $U_{s} \in \mathcal{U}_{d}^{s}, 0 \leq s \leq d-1$, such that

$$
E_{d}\left(U_{s}|g><g| U_{s}^{*}\right)=\frac{1}{d} I_{H}
$$

Let $\mathcal{A}_{d}^{s}$ be the convex set of states generated by the one-dimensional projections $|g><g|, g \in \mathcal{G}_{d}$.

Lemma 1. Given complex numbers $a, b,|a|^{2}+|b|^{2}=1$ there exist the real numbers $\phi, \psi, \alpha \in[0,2 \pi]$ such that

$$
\begin{gathered}
e^{i \phi} a+e^{i \psi} b=1, \\
e^{i \phi} a-e^{i \psi} b=e^{i \alpha} .
\end{gathered}
$$

Proof.

Pick up $\tilde{\phi}, \tilde{\psi}$ such that $\cos \tilde{\phi}=\sin \tilde{\psi}=|a|,-\sin \tilde{\phi}=\cos \tilde{\psi}=|b|$. Then, $\phi=\tilde{\psi}-\arg (a), \quad \psi=\tilde{\psi}-\arg (b), \cos \alpha=\cos (2 \tilde{\phi}), \quad \sin \alpha=$ $\sin (2 \tilde{\phi})$ give us the solution.

Proposition 1. Suppose that $d=2$. Then, $\mathcal{G}_{2}^{0}=\mathcal{G}_{2}^{1}=H$ and, hence, $\mathcal{A}_{2}^{0}=\mathcal{A}_{2}^{1}=\sigma(H)$.

Proof.

We shall prove that $\mathcal{G}_{2}^{0}=H$. The identity $\mathcal{G}_{2}^{1}=H$ can be considered analogously. 
Take a vector $g \in H$. Suppose that the coordinates of $g$ in the basis $e_{0}^{0}, e_{1}^{0}$ are $<e_{0}^{0}\left|g>=a,<e_{1}^{0}\right| g>=b$. Let the numbers $\phi, \psi, \alpha \in[0,2 \pi]$ are defined by Lemma 1 . Determine the unitary operator $U \in \mathcal{U}_{2}^{0}$ by the formula

$$
U=e^{i \phi}\left|e_{0}^{0}><e_{0}^{0}\right|+e^{i \psi}\left|e_{1}^{0}><e_{1}^{0}\right|,
$$

then, it follows from Lemma 1 , that

$$
<e_{0}^{2}\left|U g>=\frac{1}{\sqrt{2}},<e_{1}^{2}\right| U g>=\frac{e^{i \alpha}}{\sqrt{2}} .
$$

Proposition 2. $\mathcal{G}_{3}^{s} \neq H, 0 \leq s \leq 2$.

Proof.

We shall prove that $\mathcal{G}_{3}^{0} \neq H$. The other cases can be considered analogously.

Take a unit vector $g \in H$ whose coordinates $\left\langle e_{j}^{0} \mid g\right\rangle=\alpha_{j}, 0 \leq$ $j \leq 2$. If $\mathcal{G}_{3}^{0}=H$, then there exist the real numbers $\phi_{j}, \beta_{j}, 0 \leq j \leq 2$, satisfying the relations

$$
\sum_{k=0}^{2} e^{i \phi_{k}} e^{i \frac{2 \pi}{3} j k}=e^{i \beta_{j}}, 0 \leq j \leq 2 .
$$

Suppose that $\alpha_{1}, \alpha_{2}>0$ and $\alpha_{3}=0$, then the condition (12) implies that

$$
\begin{gathered}
\cos \left(\phi_{1}-\phi_{2}\right)=0, \\
\cos \left(\phi_{1}-\phi_{2}-\frac{2 \pi}{3}\right)=0
\end{gathered}
$$

and

$$
\cos \left(\phi_{1}-\phi_{2}-\frac{4 \pi}{3}\right)=0
$$

But the system of these three equation has no solution.

Lemma 2. Given complex numbers $\alpha_{j}, \sum_{j=0}^{2}\left|\alpha_{j}\right|^{2}=1$ satisfying the relations

$$
\begin{aligned}
& \left|\alpha_{1} \alpha_{2}\right|+\left|\alpha_{1} \alpha_{3}\right|>\left|\alpha_{2} \alpha_{3}\right|, \\
& \left|\alpha_{1} \alpha_{2}\right|+\left|\alpha_{2} \alpha_{3}\right|>\left|\alpha_{1} \alpha_{3}\right|, \\
& \left|\alpha_{1} \alpha_{3}\right|+\left|\alpha_{2} \alpha_{3}\right|>\left|\alpha_{1} \alpha_{2}\right| .
\end{aligned}
$$


there exist the real numbers $\phi_{j}, \beta_{j}, 0 \leq j \leq 2$, such that

$$
\sum_{j=0}^{2} e^{i\left(\phi_{j}+\frac{2 \pi j k}{3}\right)} \alpha_{j}=e^{i \beta_{k}}, 0 \leq k \leq 2 .
$$

\section{Proof.}

Because $\alpha_{j}=\left|\alpha_{j}\right| e^{i a r g\left(\alpha_{j}\right)}$, it suffices to consider only the case of non-negative real numbers $\alpha_{j} \geq 0,0 \leq j \leq 2$. Denote by $\gamma_{j}, 0 \leq$ $j \leq 2$, the angles of the triangle with the sides equal to the values $\alpha_{1} \alpha_{2}, \alpha_{1} \alpha_{3}$ and $\alpha_{2} \alpha_{3}$. Then,

$$
\begin{gathered}
\frac{\sin \gamma_{1}}{\alpha_{1} \alpha_{2}}=\frac{\sin \gamma_{2}}{\alpha_{1} \alpha_{3}}=\frac{\sin \gamma_{3}}{\alpha_{2} \alpha_{3}}, \\
\alpha_{1} \alpha_{2}=\alpha_{1} \alpha_{3} \cos \left(\gamma_{3}\right)+\alpha_{2} \alpha_{3} \cos \left(\gamma_{2}\right), \\
\alpha_{1} \alpha_{3}=\alpha_{1} \alpha_{2} \cos \left(\gamma_{3}\right)+\alpha_{2} \alpha_{3} \cos \left(\gamma_{1}\right), \\
\alpha_{2} \alpha_{3}=\alpha_{1} \alpha_{2} \cos \left(\gamma_{2}\right)+\alpha_{1} \alpha_{3} \cos \left(\gamma_{1}\right) .
\end{gathered}
$$

Put

$$
\begin{gathered}
\phi_{1}=0, \phi_{2}=\frac{2 \pi}{3}+\frac{1}{3} \gamma_{2}-\frac{1}{3} \gamma_{3}, \\
\phi_{3}=\frac{\pi}{3}-\frac{1}{3} \gamma_{2}-\frac{2}{3} \gamma_{3} .
\end{gathered}
$$

Thus, we get

$$
\begin{gathered}
\alpha_{1} \alpha_{2} \cos \left(\phi_{1}-\phi_{2}\right)+\alpha_{1} \alpha_{3} \cos \left(\phi_{1}-\phi_{3}\right)+\alpha_{2} \alpha_{3} \cos \left(\phi_{2}-\phi_{3}\right)= \\
\alpha_{1} \alpha_{2} \cos \left(\phi_{2}\right)-\alpha_{1} \alpha_{3} \cos \left(\phi_{2}+\gamma_{3}\right)-\alpha_{2} \alpha_{3} \cos \left(\phi_{2}-\gamma_{2}\right)= \\
\left(\alpha_{1} \alpha_{2}-\alpha_{1} \alpha_{3} \cos \left(\gamma_{3}\right)-\alpha_{2} \alpha_{3} \cos \left(\gamma_{2}\right)\right) \cos \left(\phi_{2}\right)+ \\
\left(\alpha_{1} \alpha_{3} \sin \left(\gamma_{3}\right)-\alpha_{2} \alpha_{3} \sin \left(\gamma_{2}\right)\right) \sin \left(\phi_{2}\right)=0
\end{gathered}
$$

due to (13) and (14). Analogously,

$$
\begin{gathered}
\alpha_{1} \alpha_{2} \sin \left(\phi_{1}-\phi_{2}\right)-\alpha_{1} \alpha_{3} \sin \left(\phi_{1}-\phi_{3}\right)+\alpha_{2} \alpha_{3} \sin \left(\phi_{2}-\phi_{3}\right)= \\
-\alpha_{1} \alpha_{2} \sin \phi_{2}+\alpha_{1} \alpha_{3} \sin \left(\phi_{2}+\gamma_{3}\right)+\alpha_{2} \alpha_{3} \sin \left(\phi_{2}-\gamma_{2}\right)= \\
\left(-\alpha_{1} \alpha_{2}+\alpha_{1} \alpha_{3} \cos \left(\gamma_{3}\right)+\alpha_{2} \alpha_{3} \cos \left(\gamma_{2}\right)\right) \sin \left(\phi_{2}\right)+ \\
\left(\alpha_{1} \alpha_{3} \sin \left(\gamma_{3}\right)-\alpha_{2} \alpha_{3} \sin \left(\gamma_{2}\right)\right) \cos \left(\phi_{2}\right)=0
\end{gathered}
$$


Denote

$$
I_{k}=\sum_{j=0}^{2} e^{i\left(\phi_{j}+\frac{2 \pi j k}{3}\right)} \alpha_{j}, 0 \leq k \leq 2 .
$$

Under the condition $\sum_{j=0}^{2} \alpha_{j}^{2}=1$ the formulas (15)-(16) imply that

$$
\operatorname{Re}\left(I_{k}\right)^{2}+\operatorname{Im}\left(I_{k}\right)^{2}=1,0 \leq k \leq 2 .
$$

Proposition 3. The set $\mathcal{G}_{3}^{s}$ includes the unit vectors $h$ whose coordinates $\alpha_{1}, \alpha_{2}$ and $\alpha_{3}$ in the basis $\left(e_{j}^{s}\right)$ satisfy the relation

$$
\begin{aligned}
& \left|\alpha_{1} \alpha_{2}\right|+\left|\alpha_{1} \alpha_{3}\right|>\left|\alpha_{2} \alpha_{3}\right|, \\
& \left|\alpha_{1} \alpha_{2}\right|+\left|\alpha_{2} \alpha_{3}\right|>\left|\alpha_{1} \alpha_{3}\right|, \\
& \left|\alpha_{1} \alpha_{3}\right|+\left|\alpha_{2} \alpha_{3}\right|>\left|\alpha_{1} \alpha_{2}\right| .
\end{aligned}
$$

Remark. We doesn't consider the trivial case $\alpha_{i}=\delta_{i i_{0}}$ for some $i_{0}, 0 \leq i_{0} \leq 2$.

Proof.

As it was done above we shall consider only the case $s=0$.

Take a vector $g$ satisfying the relations (17). Suppose that the coordinates of $g$ in the basis $e_{0}^{0}, e_{1}^{0}, e_{2}^{0}$ are $<e_{k}^{0} \mid g>=\alpha_{k}, 0 \leq k \leq 2$. Let the numbers $\phi_{j}, 0 \leq j \leq 2$, are defined by Lemma 2. Determine the unitary operator $U \in \mathcal{U}_{3}^{0}$ by the formula

$$
U=\sum_{j=0}^{2} e^{i \phi_{j}}\left|e_{j}^{0}><e_{j}^{0}\right|,
$$

then, it follows from Lemma 2 that

$$
\left|<e_{j}^{3}\right| U g>\mid=\frac{1}{\sqrt{3}}, 0 \leq j \leq 2 .
$$

Denote by $M_{d}$ and $M(0)$ the algebra of all $d \times d$ matrices and its subalgebra generated by the cyclic group $\left\{U_{m, 0}, 0 \leq n \leq d-1\right\}$, respectively. Let $\mathcal{U}_{d}$ be the maximum commutative group constructed by means of the basis $\left(e_{j}\right)_{j=0}^{d-1}$.

Proposition 4. The inclusion $W \in M(0)$ holds for all $W \in \mathcal{U}_{d}$.

Proof.

It suffices to notice that $U_{m, 0} \in \mathcal{U}_{d}, 0 \leq m \leq d-1$. 


\section{The covariant Weyl channels.}

The quantum channel $\Phi$ is said to be covariant with respect to the group $\mathcal{U}$ being a subgroup of the group of all unitary operators in $H$ if

$$
\Phi\left(U x U^{*}\right)=U \Phi(x) U^{*}, x \in \sigma(H), U \in \mathcal{U} .
$$

The Weyl operators $U_{m, n}$ satisfying the relation (7) form the basis in the algebra of all $d \times d$ matrices. Moreover, the action of a Weyl channel (9) on the Weyl operator $U_{s, t}$ is given by the formula

$$
\Phi\left(U_{s, t}\right)=\lambda_{s t} U_{s, t}
$$

where

$$
\lambda_{s t}=\sum_{m, n=0}^{d-1} \pi_{m, n} e^{2 \pi i(s n-t m) / d} .
$$

Proposition 5. Suppose that $\lambda_{s t}=\mu_{t}=$ const, $0 \leq s \leq d-1,1 \leq$ $t \leq d-1$. Then the Weyl channel is covariant with respect to the maximum commutative group $\mathcal{U}_{d}^{d}$.

Remark. If $d=2$, a role of the maximum commutative group of unitaries can be played by all multiples of the group $S O(2)$ consisting of all rotations in $H$ implemented by the matrices of the form

$$
e^{i \psi}\left(\begin{array}{cc}
\cos \phi & \sin \phi \\
-\sin \phi & \cos \phi
\end{array}\right), \phi, \psi \in[0,2 \pi]
$$

It is straightforward to check that the bistochastic qubit channel determined by the triple $\left[\lambda_{1}, \lambda_{2}, \lambda_{3}\right]$ is covariant with respect to the group of rotations iff $\lambda_{1}=\lambda_{3}$. Thus, this class includes the quantum depolarizing channel $[\lambda, \lambda, \lambda]$ and the "two-Pauli" channel $[\lambda, 1-2 \lambda, \lambda]$ as well.

Proof.

Denote $M(n)$ the algebra of matrices generated by the Weyl operators $U_{m, n}, 0 \leq m \leq d-1$. Due to Proposition 4 a unitary operator $W \in \mathcal{U}_{d}^{d}$ can be represented as

$$
W=\sum_{m=0}^{d-1} c_{m} U_{m, 0}
$$

Hence $W U_{m, 0} W^{*}=U_{m, 0}, 0 \leq m \leq d-1$, and $W M(n) W^{*} \subset M(n)$ for all $W \in \mathcal{U}_{d}^{d}, 1 \leq n \leq d-1$. It follows that if the condition of 
Proposition 5 holds, we get $W U_{s, t} W^{*} \in M(t)$ for $1 \leq t \leq d-1$. Hence,

$$
\Phi\left(W U_{s, t} W^{*}\right)=\mu_{t} W U_{s t} W^{*}=W \Phi\left(U_{s, t}\right) W^{*},
$$

$0 \leq s, t \leq d-1$.

Proposition 6. Suppose that $\pi_{m n}=p_{m}, 0 \leq m \leq d-1,1 \leq$ $n \leq d-1$. Then the Weyl channel (9) is covariant with respect to the maximum commutative group $\mathcal{U}_{d}^{d}$.

Remark. If $\pi_{00}=\left(1-\frac{d^{2}-1}{d^{2}} p\right)$ and $\pi_{m 0}=p_{n}=\frac{p}{d^{2}}, 1 \leq n, m \leq$ $d-1$, the Weyl channel (9) is the quantum depolarizing channel,

$$
\Phi(x)=(1-p) x+\frac{p}{d} \operatorname{Tr}(x), 0 \leq p \leq \frac{d^{2}}{d^{2}-1} .
$$

Suppose that $d=2$, then the "two Pauli" channel

$$
\Phi(\rho)=(1-2 p) \rho+p \sigma_{y} \rho \sigma_{y}+p \sigma_{z} \rho \sigma_{z}, \rho \in \sigma(H),
$$

is satisfied the conditions of Proposition 6 if we identify the Weyl operators with the Pauli operators such that $U_{0,0} \equiv I_{H}, U_{1,0} \equiv \sigma_{x}, U_{0,1} \equiv$ $\sigma_{y}, U_{1,1} \equiv i \sigma_{z}$.

Proof.

In the case,

$$
\begin{gathered}
\lambda_{s t}=\sum_{m=0}^{d-1} \pi_{m 0} e^{-2 \pi i t m / d}+\sum_{n=1}^{d-1} \sum_{m=0}^{d-1} p_{m} e^{2 \pi i(s n-t m) / d}= \\
\sum_{m=0}^{d-1}\left(\pi_{m 0}-p_{m}\right) e^{-2 \pi i t m / d} \equiv \mu_{t},
\end{gathered}
$$

$0 \leq s \leq d-1,1 \leq t \leq d-1$. So, the result follows from Proposition 5 .

Fix the positive numbers $0 \leq p_{n} \leq 1,0 \leq r_{m} \leq 1,1 \leq n \leq$ $d-1,0 \leq m \leq d-1$, such that $d \sum_{n=1}^{d-1} p_{n}+\sum_{m=0}^{d-1} r_{m}=1$ and consider the Weyl channel

$$
\Phi(x)=\sum_{m=0}^{d-1} r_{m} U_{m, 0} x U_{m, 0}^{*}+\sum_{m=0}^{d-1} \sum_{n=1}^{d-1} p_{n} U_{m, n} x U_{m, n}^{*},
$$

$x \in \sigma(H)$. 
Proposition 7. Suppose that d is a prime number, then the Weyl channel (19) can be represented in the form

$$
\Phi(x)=\sum_{k=0}^{d-1} \sum_{m=0}^{d-1} c_{m} U_{m, 0} \Phi_{k}(x) U_{m, 0}^{*},
$$

where

$$
\Phi_{k}(x)=\sum_{n=0}^{d-1} \lambda_{n} U_{n k \bmod d, n} x U_{n k \bmod d, n}^{*}, x \in \sigma(H),
$$

are the phase damping channels and

$$
\begin{gathered}
\lambda_{0}=1-d \sum_{n=1}^{d-1} p_{n}, \lambda_{n}=d p_{n}, 1 \leq n \leq d-1, \\
c_{m}=\frac{r_{m}}{d\left(1-d \sum_{n=1}^{d-1} p_{n}\right)}, 0 \leq m \leq d-1 .
\end{gathered}
$$

Remark. Suppose that $d=2$, then the qubit Weyl channel (19) has the following form,

$$
\Phi(\rho)=r_{0} \rho+r_{1} \sigma_{x} \rho \sigma_{x}+p_{1} \sigma_{y} \rho \sigma_{y}+p_{1} \sigma_{z} \rho \sigma_{z}, \rho \in \sigma(H),
$$

where we have identified the Weyl operators with the Pauli matrices such that $U_{0,0} \equiv I_{H}, U_{1,0} \equiv \sigma_{x}, U_{0,1} \equiv \sigma_{y}, U_{1,1} \equiv i \sigma_{z}$. In the case, in the representation (20) we get

$$
\begin{gathered}
\Phi(\rho)=\frac{r_{0}}{2\left(1-2 p_{1}\right)}\left(\Phi_{0}(\rho)+\Phi_{1}(\rho)\right)+\frac{r_{1}}{2\left(1-2 p_{1}\right)} \sigma_{x}\left(\Phi_{0}(\rho)+\Phi_{1}(\rho)\right) \sigma_{x} \\
\Phi_{0}(\rho)=\left(1-2 p_{1}\right) \rho+2 p_{1} \sigma_{y} \rho \sigma_{y}, \\
\Phi_{1}(\rho)=\left(1-2 p_{1}\right) \rho+2 p_{1} \sigma_{z} \rho \sigma_{z},
\end{gathered}
$$

$\rho \in \sigma(H)$.

\section{Proof.}

Let us compare the equations (19) and (20). In (20) it is included $d$ terms $c_{0} \lambda_{0} x, d$ terms $c_{m} \lambda_{0} U_{m, 0} x U_{m, 0}^{*}$ as well as $d$ terms

$$
c_{m-n k \bmod d} \lambda_{n} U_{m, n} x U_{m, n}^{*}, n \neq 0,
$$


(corresponding to each the channel $\Phi_{k}$ ). Hence,

$$
\begin{aligned}
d c_{m} \lambda_{0} & =r_{m}, 0 \leq m \leq d-1 \\
\sum_{k=0}^{d-1} c_{m-n k \bmod d} \lambda_{n} & =\sum_{k=0}^{d-1} c_{k} \lambda_{n}=d p_{n}, 1 \leq n \leq d-1 .
\end{aligned}
$$

Then, let us claim $\sum_{n=0}^{d-1} \lambda_{n}=\sum_{n=0}^{d-1} c_{n}=1$. It follows that

$$
\begin{gathered}
d c_{m} \lambda_{0}=r_{m}, 0 \leq m \leq d-1, \\
\lambda_{n}=d p_{n}, \quad 1 \leq n \leq d-1 .
\end{gathered}
$$

Hence,

$$
\begin{gathered}
\lambda_{0}=1-d \sum_{n=1}^{d-1} p_{n}, \\
c_{m}=\frac{r_{m}}{d\left(1-d \sum_{n=1}^{d-1} p_{n}\right)}, 0 \leq m \leq d-1 .
\end{gathered}
$$

\section{The estimation of the output entropy.}

Using Theorem 1 and Propositions 7 we can prove Theorem 2.

\section{Proof of Theorem 2.}

For $x \in \sigma(H \otimes K)$ choose the unitary operator $W$ in $H$ such that $\operatorname{Tr}_{K}(y)=W \operatorname{Tr}_{K}(x) W^{*} \in \mathcal{A}_{\text {fix }}$, where $y=\left(W \otimes I_{K}\right) x\left(W^{*} \otimes I_{K}\right)$. Here we denoted $\mathcal{A}_{\text {fix }}$ the algebra of elements being fixed with respect to the action of the group $\mathcal{U}_{d}^{d}$. The algebra $\mathcal{A}_{\text {fix }}$ is generated by the projections $\left|e_{j}^{d}><e_{j}^{d}\right|$. Hence,

$$
E_{s}\left(\operatorname{Tr}_{K}(y)\right)=\frac{1}{d} I_{H}, 0 \leq s \leq d-1,
$$

because the bases $\left(e_{j}^{s}\right)$ are mutually unbiased. Using the covariance of $\Phi$ with respect to the group of all unitary operators in $H$ we get

$$
S((\Phi \otimes I d)(x))=S((\Phi \otimes I d)(y)) .
$$


Due to Proposition 7 the tensor product of the quantum depolarizing channel and the identity channel can be represented as follows

$$
(\Phi \otimes I d)(y)=\sum_{k=0}^{d-1} \sum_{m=0}^{d-1} c_{m}\left(U_{m, 0} \otimes I_{K}\right)\left(\Phi_{k} \otimes I d\right)(y)\left(U_{m, 0}^{*} \otimes I_{K}\right),
$$

where

$$
\begin{gathered}
\left(\Phi_{k} \otimes I d\right)(y)=\sum_{n=0}^{d-1} \lambda_{n}\left(U_{n k \bmod d, n} \otimes I_{K}\right) y\left(U_{n k \bmod d, n}^{*} \otimes I_{K}\right), \\
\lambda_{0}=1-\frac{d-1}{d} p, \lambda_{n}=\frac{p}{d}, 1 \leq n \leq d-1, \\
c_{0}=\frac{1-\frac{d^{2}-1}{d^{2}} p}{d\left(1-\frac{d-1}{d} p\right)}, \\
c_{m}=\frac{p}{d^{3}\left(1-\frac{d-1}{d} p\right)}, 1 \leq m \leq d-1 .
\end{gathered}
$$

Applying Theorem 1 to each term $\left(\Phi_{k} \otimes I d\right)(y)$ included in the sum (21) we obtain the estimation (3) with $x_{j}^{s}=d \operatorname{Tr}\left(\left(\left|f_{j}^{s}><f_{j}^{s}\right| \otimes I_{K}\right) x\right)$, where $\left|f_{j}^{s}>=W^{*}\right| e_{j}^{s}>, 0 \leq j, s \leq d-1$.

Now consider the qubit case $d=2$. Let us involve the Pauli operators such that

$$
U_{0,0} \equiv I_{H}, U_{1,0} \equiv \sigma_{x}, U_{0,1} \equiv \sigma_{y}, U_{1,1} \equiv i \sigma_{z} .
$$

Then, the maximum commutative groups $\mathcal{U}_{2}^{0} \equiv \mathcal{U}_{y}, \mathcal{U}_{2}^{1} \equiv \mathcal{U}_{z}, \mathcal{U}_{2}^{2} \equiv \mathcal{U}_{x}$ are generated by the spectral projections of the Pauli operators $\sigma_{y}, \sigma_{z}$ and $\sigma_{x}$, respectively. In the following we shall also use the notation $e_{j}^{0}=e_{j}^{y}, e_{j}^{1}=e_{j}^{z}$ and $e_{j}^{2}=e_{j}^{x}, j=0,1$, as well as $E_{0}=E_{y}, E_{1}=$ $E_{z}, E_{2}=E_{x}$ for the corresponding conditional expectations.

The "two-Pauli" channel

$$
\Phi(\rho)=(1-2 p) \rho+p \sigma_{y} \rho \sigma_{y}+p \sigma_{z} \rho \sigma_{z}, \rho \in \sigma(H), 0<p<\frac{1}{2} .
$$

is covariant with respect to $\mathcal{U}_{x}$ by means of Proposition 6 .

Proposition 8. The channel (22) can be represented as a convex combination of the form

$$
\Phi(\rho)=\frac{1-3 p}{1-p} \Phi_{1}(\rho)+\frac{2 p}{1-p} \sigma_{z} \Psi_{1}(\rho) \sigma_{z},
$$


where

$$
\Phi_{1}(\rho)=(1-p) \rho+p \sigma_{y} \rho \sigma_{y}
$$

is the phase damping and the channel

$$
\Psi_{1}(\rho)=p \rho+\frac{1-p}{2} \sigma_{x} \rho \sigma_{x}+\frac{1-p}{2} \sigma_{z} \rho \sigma_{z}
$$

is covariant with respect to $\mathcal{U}_{y}$.

\section{Proof.}

It is straightforward to check the validity of the formula. To show that the channel $\Psi_{1}$ is covariant with respect to $\mathcal{U}_{y}$ let us redefine the correspondence between the Pauli operators and the Weyl operators such that

$$
\sigma_{y} \equiv U_{1,0}, \sigma_{z} \equiv U_{0,1}, \sigma_{x} \equiv-i U_{1,1} .
$$

Then, the result follows from Proposition 6 .

In the proof of Theorem 3 we shall need the "two Pauli" channel of the form

$$
\Psi_{1}(\rho)=p \rho+\frac{1-p}{2} \sigma_{x} \rho \sigma_{x}+\frac{1-p}{2} \sigma_{z} \rho \sigma_{z}, \rho \in \sigma(H) .
$$

It is straightforward to check that the following estimation holds.

Proposition 9. Suppose that $p \leq \frac{1}{3}$. Then, the channel (23) is a convex combination of the form

$$
\begin{gathered}
\Psi_{0}(\rho)=\frac{p}{1-p} \Phi_{0}(\rho)+\frac{1-3 p}{2(1-2 p)} \sigma_{x} \Phi_{1}(\rho) \sigma_{x}+ \\
\left(1-\frac{p}{1-p}-\frac{1-3 p}{2(1-2 p)}\right) \sigma_{z} \Phi_{1}(\rho) \sigma_{z}
\end{gathered}
$$

where

$$
\begin{aligned}
& \Phi_{0}(\rho)=(1-p) \rho+p \sigma_{x} \rho \sigma_{x}, \\
& \Phi_{1}(\rho)=(1-p) \rho+p \sigma_{y} \rho \sigma_{y},
\end{aligned}
$$

$\rho \in \sigma(H)$, are two phase damping channels.

\section{Proof of Theorem 3.}

Fix the state $\rho \in \sigma(H \otimes K)$. Due to Proposition 1 one can find the unitary operator $W \in \mathcal{U}_{x}$ such that $\operatorname{Tr}_{K}(\tilde{\rho})=W \operatorname{Tr}_{K}(\rho) W^{*}$ satisfies the identity

$$
E_{y}\left(\operatorname{Tr}_{K}(\tilde{\rho})\right)=\frac{1}{2} I_{H}
$$


where $\tilde{\rho}=\left(W \otimes I_{K}\right) \rho\left(W^{*} \otimes I_{K}\right)$. Using the covariance of the channel $\Phi$ with respect to $\mathcal{U}_{x}$ we obtain

$$
S((\Phi \otimes I d)(\rho))=S((\Phi \otimes I d)(\tilde{\rho})) .
$$

It follows from Proposition 8 that

$$
(\Phi \otimes I d)(\tilde{\rho})=\frac{1-3 p}{1-p}\left(\Phi_{1} \otimes I d\right)(\tilde{\rho})+\frac{2 p}{1-p}\left(\sigma_{z} \otimes I_{K}\right)\left(\Psi_{1} \otimes I d\right)(\tilde{\rho})\left(\sigma_{z} \otimes I_{K}\right),
$$

where

$$
\left(\Phi_{1} \otimes I d\right)(\tilde{\rho})=(1-p) \tilde{\rho}+p\left(\sigma_{y} \otimes I_{K}\right) \tilde{\rho}\left(\sigma_{y} \otimes I_{K}\right)
$$

and

$$
\left(\Psi_{1} \otimes I d\right)(\tilde{\rho})=p \tilde{\rho}+\frac{1-p}{2}\left(\sigma_{x} \otimes I_{K}\right) \tilde{\rho}\left(\sigma_{x} \otimes I_{K}\right)+\frac{1-p}{2}\left(\sigma_{z} \otimes I_{K}\right) \tilde{\rho}\left(\sigma_{z} \otimes I_{K}\right) .
$$

Hence, we get

$$
S((\Phi \otimes I d)(\tilde{\rho})) \geq \min \left\{S\left(\Phi_{1} \otimes I d\right)(\tilde{\rho}), S\left(\left(\Psi_{1} \otimes I d\right)(\tilde{\rho})\right)\right\} .
$$

Taking into account (24) one can apply Theorem 1 to $\left(\Phi_{1} \otimes I d\right)(\tilde{\rho})$. Then,

$$
\begin{gathered}
S\left(\left(\Phi_{1} \otimes I d\right)(\tilde{\rho})\right) \geq-(1-p) \log (1-p)-p \log p+\frac{1}{2}\left(S\left(2 \operatorname{Tr}_{H}\left(\left(\left|e_{1}^{1}><e_{1}^{1}\right| \otimes I_{K}\right) \rho\right)\right)+\right. \\
S\left(2 \operatorname{Tr}_{H}\left(\left(\left|e_{2}^{1}><e_{2}^{1}\right| \otimes I_{K}\right) \rho\right)\right),
\end{gathered}
$$

where $\left|e_{1}^{1}>=W^{*}\right| e_{0}^{y}>,\left|e_{2}^{1}>=W^{*}\right| e_{1}^{y}>$.

It follows from Proposition 1 that there exists the unitary operator $\tilde{W} \in \mathcal{U}_{y}$ such that $\operatorname{Tr}_{K}(\tilde{\tilde{\rho}})=\tilde{W} \operatorname{Tr}_{K}(\tilde{\rho}) \tilde{W}^{*}$ satisfies the identity

$$
E_{x}\left(\operatorname{Tr}_{K}(\tilde{\tilde{\rho}})\right)=\frac{1}{2} I_{H}
$$

where $\tilde{\tilde{\rho}}=\left(\tilde{W} \otimes I_{K}\right) \tilde{\rho}\left(\tilde{W}^{*} \otimes I_{K}\right)$. Moreover,

$$
E_{y}\left(\operatorname{Tr}_{K}(\tilde{\tilde{\rho}})\right)=E_{y}\left(\tilde{W} \operatorname{Tr}_{K}(\tilde{\rho}) \tilde{W}^{*}\right)=E_{y}\left(\operatorname{Tr}_{K}(\tilde{\rho})\right)=\frac{1}{2} I_{H}
$$

because $\tilde{W}$ belongs to the algebra generated by the projections $\mid e_{0}^{y}><$ $e_{0}^{y}|,| e_{1}^{y}><e_{1}^{y} \mid$, such that $E_{y}(\tilde{W})=\tilde{W}$.

The covariance of $\Psi_{1}$ with respect to $\mathcal{U}_{y}$ gives us

$$
S\left(\left(\Psi_{1} \otimes I d\right)(\tilde{\rho})\right)=S\left(\left(\Psi_{1} \otimes I d\right)(\tilde{\tilde{\rho}})\right) .
$$


It follows from Proposition 9 that the channel $\Psi_{1} \otimes I d$ can be represented in the form

$$
\begin{gathered}
\left(\Psi_{1} \otimes I d\right)(\tilde{\tilde{\rho}})=\frac{p}{1-p}\left(\Phi_{0} \otimes I d\right)(\tilde{\tilde{\rho}})+\frac{1-3 p}{2(1-2 p)}\left(\sigma_{x} \otimes I_{K}\right)\left(\Phi_{1} \otimes I d\right)(\tilde{\tilde{\rho}})\left(\sigma_{x} \otimes I_{K}\right)+ \\
\left(1-\frac{p}{1-p}-\frac{1-3 p}{2(1-2 p)}\right)\left(\sigma_{z} \otimes I_{K}\right)\left(\Phi_{1} \otimes I d\right)(\tilde{\tilde{\rho}})\left(\sigma_{z} \otimes I_{K}\right)
\end{gathered}
$$

where

$$
\begin{aligned}
& \left(\Phi_{0} \otimes I d\right)(\tilde{\tilde{\rho}})=(1-p) \tilde{\tilde{\rho}}+p\left(\sigma_{x} \otimes I_{K}\right) \tilde{\tilde{\rho}}\left(\sigma_{x} \otimes I_{K}\right), \\
& \left(\Phi_{1} \otimes I d\right)(\tilde{\tilde{\rho}})=(1-p) \tilde{\tilde{\rho}}+p\left(\sigma_{y} \otimes I_{K}\right) \tilde{\tilde{\rho}}\left(\sigma_{y} \otimes I_{K}\right),
\end{aligned}
$$

Taking into account the identities (27) and (28) one can apply Theorem 1 to $\left(\Phi_{0} \otimes I d\right)(\tilde{\tilde{\rho}})$ and $\left(\Phi_{1} \otimes I d\right)(\tilde{\tilde{\rho}})$. Thus, we obtain

$$
\begin{gathered}
S\left(\left(\Phi_{0} \otimes I d\right)(\tilde{\tilde{\rho}})\right) \geq-(1-p) \log (1-p)-p \log p+\frac{1}{2}\left(S\left(2 \operatorname{Tr}_{H}\left(\left(\left|e_{1}^{2}><e_{1}^{2}\right| \otimes I_{K}\right) \rho\right)\right)+\right. \\
\left.S\left(2 \operatorname{Tr}_{H}\left(\left(\left|e_{2}^{2}><e_{2}^{2}\right| \otimes I_{K}\right) \rho\right)\right)\right),
\end{gathered}
$$

where $\left|e_{1}^{2}>=W^{*} \tilde{W}^{*}\right| e_{0}^{x}>,\left|e_{2}^{2}>=W^{*} \tilde{W}^{*}\right| e_{1}^{x}>$ and

$$
\begin{gathered}
S\left(\left(\Phi_{1} \otimes I d\right)(\tilde{\tilde{\rho}})\right) \geq-(1-p) \log (1-p)-p \log p+\frac{1}{2}\left(S\left(2 \operatorname{Tr}_{H}\left(\left(\left|e_{1}^{3}><e_{1}^{3}\right| \otimes I_{K}\right) \rho\right)\right)+\right. \\
\left.S\left(2 T r_{H}\left(\left(\left|e_{2}^{3}><e_{2}^{3}\right| \otimes I_{K}\right) \rho\right)\right)\right),
\end{gathered}
$$

where $\left|e_{1}^{3}\right\rangle=W^{*} \tilde{W}^{*}\left|e_{0}^{y}>,\right| e_{2}^{3}>=W^{*} \tilde{W}^{*} \mid e_{1}^{y}>$. Combining (25), (26), (29), (30) and (31) we obtain the result.

\section{Acknowledgments}

The author is grateful to Mary Beth Ruskai for the remark about the "two-Pauli" channel and sending a draft of her unpublished manuscript which partially inspired this work. This paper would not be possible without a detailed report on its first version by the anonymous referee I like to thank especially. 


\section{References}

[1] Amosov G.G., Holevo A.S., Werner R.F. On some additivity problems in quantum information theory. Probl. Inf. Transm. 2000. V. 36. N 4. P. 24-34; LANL e-print quant-ph/0003002

[2] Amosov G.G. Remark on the additivity conjecture for the depolarizing quantum channel. Probl. Inf. Transm. 42 (2006) 3-11. LANL e-print quant-ph/0408004

[3] Datta N., Holevo A.S., Suhov Y. Additivity for transpose depolarizing channels. Probl. Inf. Transm. 41 (2005) 76-90. LANL e-print quant-ph/0412034.

[4] Datta N, Ruskai M.B. Maximal output purity and capacity for asymmetric unital qudit channels J. Physics A: Mathematical and General 38 (2005) 9785-9802. LANL e-print quant-ph/0505048

[5] Fannes M., Haegeman B., Mosonyi M., Vanpeteghem D. Additivity of minimal entropy output for a class of covariant channels. LANL e-print quant-ph/0410195

[6] Fivel D.I. Remarkable Phase Oscillations Appearing in the Lattice Dynamics of Einstein-Podolsky-Rosen States. Phys. Rev. Lett. 74 (1995) 835-838.

[7] Fukuda M., Holevo A.S. On Weyl-covariant channels. LANL eprint quant-ph/0510148.

[8] Holevo A.S. On the mathematical theory of quantum communication channels. Probl. Inf. Transm. 8 (1972) 62 - 71.

[9] Holevo A.S. Quantum coding theorems. Russ. Math. Surveys 53 (1998) 1295-1331; LANL e-print quant-ph/9808023.

[10] Holevo A.S. Additivity conjecture and covariant channels. Int. J. Quantum Inf. 3 (2005) 41-47 (2005).

[11] Ivanovich I.D. Geometrical description of quantum state determination. J. Physics A 14 (1981) 3241-3245.

[12] Karpov E., Daems D., Cerf N.J. Entanglement enhanced classical capacity of quantum communication channels with correlated noise in arbitrary dimensions. LANL e-print quant-ph/0603286.

[13] King C. Additivity for unital qubit channels. J. Math. Phys. 43 (2002) 4641-4653; LANL e-print quant-ph/0103156. 
[14] King C. The capacity of the quantum depolarizing channel. IEEE Trans. Inform. Theory 49 (2003) 221-229; LANL e-print quant-ph/0204172

[15] Lindblad G. Completely positive maps and entropy inequalities. Commun. Math. Phys. 40 (1975) 147-151.

[16] Shor P. Additivity of the classical capacity of entanlementbreaking quantum channels. J. Math. Phys. 43 (2002) 4334-4340. LANL e-print quant-ph/0201149

[17] Shor P. Equivalence of additivity questions in quantum information theory. Comm. Math. Phys. 246 (2004), no. 3, 453-472.

[18] Umegaki H. Conditional expectation in an operator algebra. IV. Entropy and information. Kodai Math. Sem. Rep. 14 (1962) 5985 . 